\title{
TPM 在粮油仓储管理中的应用探讨
}

\author{
丁孝银
}

河南工业大学 河南省粮油仓储建筑与安全重点实验室

DOI:10.18686/bd.v2i3.1288

[摘要] 随着如今粮油生产各项技术的不断提高, 对粮油仓储的安全管理也有了更高的要求。本文主要论述了 TPM 活动 在根油仓储中的应用,并分点讨论了该管理技术对储存工序的重要意义,希望通过该管理体制的持续深入为我国粮油仓储 的现场管理提供助力,实现安全管理,高效生产的目标。

[关键词] 粮油仓储;TPM; 现场管理; 安全

\section{1 简述 TPM 技术}

在粮油的仓储管理当中,TPM 技术就是指全员性的生 产保全活动,该技术在 1971 年被首次提出。这里的全员性 泛指参加生产的全体员工,包含生产公司层人员、车间负责 生产人员以及办公室管理人员, 以上提到的人员将全部加 人到该生产活动的日常管理与养护任务当中。随着时代的 进步, 各项新技术不断融入与应用,TPM 的工作内容也相应 得到了扩充, 进行了全方位的转换与升级。到了 1989 年, 该 技术完成内容革新, 其最终目的就是为了达到全方位、立体 式的高效率生产构建高水准生产系统, 力在以该体系为立 足点减少各生产环节发生故障的概率。其中故障包括环境 意外灾害以及机器故障等事故, 该管理体系涵盖了第一生 产线、生产设计、技术开发、产品销售以及生产管理等部门, 意在将该生产管理贯彻到企业的每个层级之中, 做到高效 率、高质量、低事故、低损耗。

\section{2 在粮油的仓储管理中应用 TPM}

我国的平均粮油储存量极大, 生产设备样式繁多, 且粮 油制备过程涉及专业知识较多, 操作难度大。负责一线生产 的人员既要做好生产粮油的品质保障, 又要做好粮油仓储 管理的维护, 这样才能使得粮油在生产及后期存储过程中 无事故发生, 不给企业带来经济损失, 确保各条生产线的顺 利运。所以在实际生产管理过程中, 需要工作人员具有良好 的专业素质, 对粮油的仓储管理具有一定的知识积累与经 验, 这就增强了该项工作的难度。由于 TPM 技术力在突出 企业全体人员的加人与管理, 在粮油仓储中融入该技术, 可 以形成一整套粮油生产监管系统。其重点特色就是让全员 均投人到各自掌管的工作任务之中, 利用集体的力量克服 生产及储备过程中的各项技术难题, 以及后期的设备养护、 车间清理当中, 保证粮油的储存管理能够在干净整洁的环 境中进行。再加上粮油生产设施的有效运行, 减少了因机器 故障带来的突发事故, 避免企业的经济损失。此外, 将该技术 引人到粮油仓储当中, 还能够加强工作人员的工作品质, 员 工可以自觉发现生产中存在的问题并动用自己的力量予以 解决,及时防止有害生产的事故发生。

\section{3 在粮油的仓储管理中应用 TPM 的主要项目}

3.1 TPM 活动在仓储现场的清理工作中的应用

引人该活动实现员工对粮油仓储过程中的现场管理与 清洁工作。通过这种现场管理,能够让工作人员养成较好的 工作习惯,为生产现场提供最为有利的储存条件, 保证企业 的利益最大化。员工通过对仓储现场的整顿管理, 能够清理 出现场的工业生产废物, 并将仓储中经常用到的器具按照 一定规律进行分类并整齐放置。同时,工作人员对现场的清 理工作能够改善粮油的仓储环境, 保证仓储的卫生满足生 产相关标准规范, 严格制度化的现场监管还可以让工作人 员形成良好的工作习惯, 自主自觉的保护储存现场的环境, 营造干净整洁的工作氛围, 为后续的储存环节提供最佳工 作基础。另外,由于工作人员对仓储现场的维护与管理,使得 各生产器具均正确放置在各自的位置上, 一旦员工有需要 就可在固定位置找到器具, 保证了生产的连贯性。这种井然 有序的放置大大提高了员工的工作效率, 使整个粮油生产 储存环节有序进行。并且, 通过上述对工作现场的管理, 还可 加强员工的工作素质,为粮油仓储提供坚实的基础保障。

3.2 去除危害源的不利影响

为了有效规避危害源的影响, 粮油企业应该加强对六 源的管理防治力度, 在整个生产过程中号召所有工作人员 进行六源的治理工作。通过全方位的排查检测,找到生产过 程中的危害源头或安全隐患, 及时针对危害问题提出解决 方案,减少危害源给粮油储存带来的不利影响。因为上面提 到的六源, 就是引发各种生产事故的内在起因, 所以及时检 测出六源并加以防治对粮油的存储工作具有极其重要的维 护作用。比如,如果对浅圆仓的皮带机不定时清理养护, 就会 因表面粉尘过多进而发生自燃的现象, 降低粮油储存的安 全机制。而利用 TPM 活动, 增强危害源的检查力度, 定期对 生产设备进行清理养护工作, 粉尘的及时清扫避免了自燃 等事故的发生,提高了粮油储存的安全系数。

3.3 TPM 活动对储存方案的提升

将该活动引人粮油储存工序中, 能够动用员工的自我 创新能力进而提出行之有效的改进方案, 完善粮油存储过 程。利用集体性的创新思维提高存储管理质量, 减少企业产 生不必要的资源损耗。此外, 粮油企业还可以设立明确的奖 
励制度, 对工作中表现突出且对粮油仓储管理提出建设性 意见的员工予以奖励, 这样可以增强员工的工作积极性, 促 进管理品质的提升。

3.4 提高粮油储存的安全性管理

由于粮油的存储现场涉及对象众多, 环境较为复杂多 变,所以危害源的分布也较为分散不易发现。再加上如今粮 油生产的各类设备繁多, 因此对粮油储存现场实行周密严 格的安全性管理很有必要。引人 TPM 活动的目视化管理, 就是在粮油存储现场标记出指引明确的警戒线, 对存在危 害源的部分做出危险警告标志, 并且对粮油仓储涉及的重 要生产设施设定注意事项的指示说明、操作工序以及后期 维护任务等等, 将上述指引标记贴在生产现场较为醒目的 位置,发挥出警示指导功能,提高存储现场的安全性。可以 说,应用目视化的现场管理, 就是让粮油存储现场中的危害 源完全呈现出来, 参与生产的所有员工均能够了解危害源 的所在, 适时规避预防, 有助于仓储过程安全化生产的进行。

\section{TPM 活动在粮油储存工序中的实际作用成效}

\section{1 危害源的检查获得成效}

随着 TPM 的持续引入, 对存储现场危害源的检查力度 加大,危害源被准确查出并采取相应措施加以解决, 使得现 场的安全性有了一定保障,危害源的预防和解决获得成效。 具体来说, 就是在实际粮油仓储现场中, 库管主人以及分管 领导带领员工对存储现场、设施运行状况以及办公用品等 多方面开展危害源的排查工作,发现危害源及时记录、归纳 整理,并上报给相应管理层,按照危害源的种类提出有针对 性的解决策略。与此同时,TPM 专员以月为单位对各类危害 源按照影响对象及危害程度进行种类划分, 并制定相应改 进对策, 部分需要集体合作或者仅需单人完成的危害源,则 由生产班长或者个人负责问题的处理, 如果危害源过于复 杂, 解决难度较大, 则需要由 TPM 专业人员进行协助, 克服 技术难题。

4.2 储存现场相应警示标识的明确

在没有开展 TPM 管理以前,现场安装、悬挂了不少的 安全警示标志、标识,但是这些标识、标志多是员工自己设 计的,没有系统性,有些标志甚至不符合安全管理标志、标识 规范要求。针对这个问题,员工通过对日常工作容易出现危 险或者需要强调小心的位置,如上下仓顶的爬梯、输送设备 的重力张紧装置、输油管道的进出方向、参观通道和生产作 业通道无区分、关键设备的操作流程未上墙等进行统计分 析。然后,按照安全管理标志、标识规范与要求,重新设计制
作和张贴、悬挂安全警示标志或者标识、关键操作步骤上 墙、划分人员通道、标识管道流向等使其规范统一。在这个 过程中不仅使员工的安全知识得到了拓展, 更使安全意识 得到了强化。

4.3 粮油仓储库房的安全管理得到完善

在实行 TPM 管理以前, 库房由于机械设备、备品、备 件、工具等种类繁多再加上淘汰、废弃物资的堆放,导致拥 挤不堪, 不能很好地利用库房空间,降低了查找和领用设备 工具的效率。实行 TPM 管理以后, 首先, 对库房的功能区域 进行了重新划分,按照机械设备的类型分为谷冷机区、制氮 机区、螺杆泵区、叉车区、装载车区、登高车区、货车区,并根 据设备的使用频率安排存放区域; 其次,对无法再利用的废 弃物资按照直属库规定整理后交综合部门统一处理，提高 库房空间的利用率; 第三,设置工具区。对日常保管工具涉 及的 8 个大类进行分类存放,并设立单独的领用、借用记录, 方便工具设备的查找和追溯; 第四,对所有规划的小区域按 照使用频率分级别用不同颜色的线进行定位, 同时在库房 设置一块临时区域供科研实验、维修改造材料的临时堆放, 对所有设备的朝向、摆放高度、位置、标识等进行规范和统 一,整个库房的有效面积得到了高效利用,自然大大提高了 查找和取用归还设备的效率。

\section{5 结束语}

综上所述,在粮油的仓储管理中引人 TPM 活动, 可以极 大程度地改善仓储现场的安全管理以及专业人员的工作素 养,通过运用 TPM 各项功能管理,全面查找存储现场危害源 所在,进而提出预防对策,减少现场事故的发生。另外,TPM 的管理方法, 还可以将生产现场存在的危险部分进行明确 的标记工作,让各危险源暴露于表面,员工均可以对警示标 识进行相应的预防工作, 加强存储现场环境及生产设备的 安全维护。所以在现代化的粮油存储中融人 TPM 技术是保 证存储安全、各项生产活动顺利运行的基础管理保障,同时 也是推动当今粮油生产行业稳步运行的最佳动力。

\section{参考文献:}

[1]郝立群,赵旭.加强粮食仓储业安全生产工作确保国 家储粮安全[J].粮食加工,2014,39(04):68-70.

[2]王朝强。粮油仓储管理工作的有效对策 [J]. 食品安 全导刊,2016,(27):90.

[3]刘永利.推广绿色储粮技术提高粮油仓储综合效益 [J].粮油仓储科技通讯,2017,33(01):3-5. 